\title{
Modelo de ecuaciones estructurales para predecir el bienestar y la dependencia funcional en adultos mayores de la República Dominicana
}

\author{
Amparo Oliver, ${ }^{1}$ Esperanza Navarro, ${ }^{2}$ Juan Carlos Meléndez, ${ }^{2}$ \\ Cristina Molina ${ }^{3}$ y José Manuel Tomás ${ }^{1}$
}

Forma de citar Oliver A, Navarro E, Meléndez JC, Molina C, Tomás JM. Modelo de ecuaciones estructurales para predecir el bienestar y la dependencia funcional en adultos mayores de la República Dominicana. Rev Panam Salud Publica. 2009;26(3):189-96.

RESUMEN Objetivo. Generar un modelo estructural multifactorial que explique la dependencia funcional y el bienestar a partir de algunas características sociodemográficas asociadas con esos dominios, en adultos mayores de la República Dominicana.

Métodos. Estudio transversal mediante encuesta en una muestra de 1397 personas de 65 años o más representativa de la población de adultos mayores de la República Dominicana. Se recabó información sociodemográfica de los entrevistados (sexo, edad, nivel de estudios, si realizaba alguna actividad remunerada y nivel de ingresos) y los datos necesarios para calcular el indice de Barthel y las respuestas a las preguntas de las escalas del bienestar psicológico de Ryff. Para identificar las relaciones entre las variables de interés se probaron tres modelos de ecuaciones estructurales.

Resultados. El modelo estructural de ajuste más satisfactorio relacionó las características sociodemográficas con la capacidad para realizar las actividades básicas de la vida diaria $(A B V D)$ y las dimensiones del bienestar psicológico de las escalas de Ryff sin proponer factores latentes. La capacidad predictiva del modelo para las variables endógenas fue débil. La edad se relacionó negativamente con algunas dimensiones del bienestar y con la capacidad para realizar las $A B V D$; el sexo se relacionó con la dimensión de relaciones positivas con otros; y el nivel de estudios influyó positivamente sobre el dominio del ambiente, el crecimiento personal y el propósito en la vida, así como en la capacidad para realizar las ABVD.

Conclusiones. Los modelos validados brindan información necesaria para desarrollar politicas orientadas a dos niveles de acción complementarios: la promoción de la autonomía de los adultos mayores y el aumento de la cobertura de los sistemas de enseñanza formal. Estos resultados respaldan la conveniencia de aumentar las inversiones en politicas sociales.

Palabras clave Salud del anciano; calidad de vida; modelos matemáticos; República Dominicana.

Departamento de Metodología de las Ciencias del Comportamiento, Universidad de Valencia, Valencia, España. La correspondencia se debe dirigir a Amparo Oliver, Departamento de Metodología de las Ciencias del Comportamiento, Universidad de Valencia, Av. Blasco Ibáñez No. 21, Valencia 46010, España. Correo electrónico: oliver@uv.es, tomasjm@uv.es

2 Departamento de Psicología Evolutiva y de la Educación, Universidad de Valencia, Valencia, España.

3 Universidad Autónoma de Santo Domingo, Santo Domingo, República Dominicana.
En América Latina y el Caribe, la población de 60 años o más aumenta sostenidamente en todos los países. Se trata de un proceso generalizado de envejecimiento de las estructuras demográficas provocado entre otros factores por el aumento de la esperanza de vida en 20 años — hasta llegar a 70 años-y la disminución de la tasa general de fecundi- dad, que pasó de alrededor de seis hijos por matrimonio a menos de tres (1). De este modo se ha producido un aumento, tanto en el número de adultos mayores como en la proporción relativa de este grupo de edad en la población total.

Así, si en 1950 había aproximadamente 10 millones de personas de 60 años o más en América Latina y el Caribe, al termi- 
nar el siglo XX esa cifra se había cuadruplicado (1) y entre los años 2000 y 2025 se incorporarán alrededor de 57 millones de adultos mayores más para alcanzar la considerable cifra de 98 millones. Se estima que entre 2025 y 2050, el incremento será de 86 millones de personas. Aunque los países de mayor población concentrarán la mayor parte de este aumento, en los países más pequeños el incremento también será significativo, especialmente a partir de 2025, ya que la población de adultos mayores crecerá en esos países en 20-25\% hasta el año 2050 (2).

Estos cambios en la estructura de la población también afectan directamente a la República Dominicana que, si bien actualmente mantiene una estructura de población joven, muestra una clara tendencia al envejecimiento. Esto se debe, sobre todo, a la reducción de la tasa de fecundidad — que ha pasado de 7,5 hijos por matrimonio en la década de 1950 a 2,73 al finalizar el milenio pasado-, las modificaciones en la esperanza de vida —que aumentó en 24 años en ese mismo período-y el descenso de la mortalidad infantil — que ha pasado de 149 a 34 por 1000 nacidos vivos-. De esta manera, si actualmente los adultos mayores de 60 años o más constituyen aproximadamente $8 \%$ de la población dominicana, en 2025 serán 12,9\% y en 2050 prácticamente duplicarán esa cifra (3).

No obstante, se debe tener en cuenta que el aumento en la esperanza de vida no significa necesariamente una mejora en las condiciones de salud (4). Al contrario, uno de los problemas más importantes en la etapa de adulto mayor es la pérdida de las capacidades funcionales, emocionales y cognoscitivas. Conocer el alcance y las circunstancias de la dependencia de los adultos mayores es importante, ya que constituye un indicador de sus condiciones de salud y una forma de analizar el bienestar general de la sociedad (5).

La evidencia empírica indica que existe una estrecha relación entre la dependencia y la edad, ya que el porcentaje de personas con limitaciones en su capacidad funcional aumenta en los adultos mayores conforme aumenta la edad $(6,7)$. Por ello no es extraño que la dependencia se vea como un problema vinculado al envejecimiento. No obstante, se debe tener presente que, aún cuando los adultos mayores sean los que con más frecuencia se ven afectados, la dependencia funcional está presente en todos los grupos de edad de la población y, por tanto, no se circunscribe a los adultos mayores.

En este sentido, los resultados de la Encuesta sobre Discapacidades, Deficiencias y Estado de Salud (EDDES-99) (8), realizada en España, muestran que a partir de la adultez se produce un punto de inflexión en el que la dependencia aumenta con la edad hasta llegar a cerca de $50 \%$ a partir de los 80 años. Con relación al género, si bien hasta los 64 años los porcentajes de dependencia son algo superiores en los hombres, a partir de los 65 años esa tendencia cambia y el porcentaje de mujeres con discapacidad aumenta considerablemente y supera al grupo de los hombres en 3,6\%, diferencia que aumenta considerablemente a partir de los 70 años hasta rebasar 6,5\%.

En el ámbito latinoamericano, la Encuesta Salud, Bienestar y Envejecimiento (SABE) es un claro ejemplo del análisis de las condiciones de salud de los adultos mayores, así como de la evaluación de las limitaciones funcionales, y a partir de diferentes análisis de sus datos se puede observar la relación que existe entre la capacidad funcional y la edad (9-11).

En 1998, el Consejo de Europa, en su Recomendación No. R(98)9 del Comité de Ministros a los Estados Miembros, definió el concepto de dependencia como la necesidad de ayuda o asistencia importante para realizar las actividades de la vida cotidiana (12). De manera más precisa se puede definir como el estado en que se encuentran las personas que por razones ligadas a la falta o la pérdida de su autonomía física, psíquica o intelectual tienen necesidad de asistencia o ayuda importante para realizar las actividades habituales de la vida diaria $y$, de modo particular, los referentes al cuidado personal. De este modo, quedan recogidas tres de las claves fundamentales de la dependencia: la existencia de una limitación, la incapacidad para realizar actividades y la necesidad de ayuda.

Esta perspectiva es coherente con lo recogido en la Clasificación Internacional del Funcionamiento de la Discapacidad y de la Salud (CIF) (13), según la cual la dependencia puede entenderse como el resultado de un proceso que se inicia con la aparición de un déficit en el funcionamiento corporal o de una función fisiológica o mental como consecuencia de una enfermedad o un accidente. Este déficit comporta una limitación en la actividad. Cuando esta limitación no se puede compensar mediante la adaptación del en- torno, provoca una restricción en la participación que se concreta en la dependencia de la ayuda de otras personas para realizar las actividades de la vida cotidiana.

Así, la independencia para realizar las actividades básicas de la vida diaria (ABVD) se convierte en un elemento fundamental del adecuado desarrollo en la vejez y forma parte del concepto de envejecimiento con éxito $(14,15)$, definido como una baja probabilidad de enfermedad y de la discapacidad asociada, un alto funcionamiento cognitivo, una adecuada funcionalidad física y un compromiso activo con la vida.

En esta misma línea, algunos autores muestran la importancia del bienestar como una variable predictora de la salud y de una adecuada capacidad funcional $(16,17)$. En una investigación que evaluó el estado de salud y emocional de 2282 mexicano-estadounidenses de 65 a 99 años se concluyó que experimentar emociones positivas protegía a esas personas de los efectos más negativos del envejecimiento y de la incapacidad (18). De este modo, parece que existe una relación directa entre el nivel de capacidad funcional y el bienestar.

En lo referente al estudio del bienestar, se han mantenido dos líneas de trabajo paralelas centradas en lo que se ha definido como bienestar psicológico y bienestar subjetivo. Ambos tipos de bienestar están relacionados, aunque cada uno posee dimensiones específicas $(19,20)$.

El bienestar psicológico se ha descrito como un esfuerzo por perfeccionarse y conseguir la realización del potencial propio, por lo que este tipo de bienestar parece estar vinculado con tener un propósito en la vida y que la vida adquiera significado para uno mismo, así como con los desafíos y un determinado esfuerzo por superarlos y conseguir metas valiosas $(19,20)$.

Por su parte, el bienestar subjetivo está más cercano a conceptos de larga tradición en la psicología y, actualmente, engloba términos como felicidad, moral y satisfacción vital. Este tipo de bienestar muestra un componente emocional o afectivo relacionado con los sentimientos de placer y displacer que experimenta la persona (felicidad) y otro componente de carácter más cognitivo referido al juicio que merece a la persona su trayectoria evolutiva (satisfacción) (21).

Al parecer existe una superposición parcial entre los dos constructos, pero las trayectorias que siguen con la edad las 
dimensiones establecidas en ellos parecen ser diferentes. Así, las dimensiones que permanecen relativamente estables son la autoaceptación (propia del bienestar subjetivo) y las relaciones positivas con los otros. La dimensión dominio del ambiente (relacionada directamente con el bienestar subjetivo) tiende a ser más alta en los adultos mayores y de mediana edad que en los jóvenes, pero permanece estable durante el paso de la mediana edad a la vejez. Un patrón similar sigue la dimensión autonomía. Por último, las dimensiones propósito en la vida y crecimiento personal, típicas del bienestar psicológico, muestran una disminución a lo largo de la vida, especialmente cuando se comparan personas de mediana edad y adultos mayores (22). La mayor parte de los estudios que relacionan la capacidad de realizar las actividades de la vida diaria y el bienestar se han realizado en países industrializados, especialmente en los anglosajones (23).

En este contexto, el objetivo del presente estudio fue generar un modelo estructural multifactorial que explique la dependencia funcional y el bienestar a partir de algunas características sociodemográficas asociadas con esos dominios, en adultos mayores de la República Dominicana.

\section{MATERIALES Y MÉTODOS}

Se realizó un estudio transversal mediante encuesta en el que participaron 1397 adultos mayores dominicanos. Para que la muestra fuera representativa de la población de adultos mayores del país se realizó un muestreo por cuotas estratificado por las 11 regiones del país y la edad. La población de 65 años en adelante se separó en cinco grupos con intervalos de cinco años y un grupo de mayores de 90 años. Se debían cumplir las cuotas de los distintos grupos de edad y región establecidas por afijación proporcional. El muestreo se diseñó para cumplir con un nivel del confianza de 95\%.

Psicólogos y orientadores de todo el país, entrenados previamente, colectaron la información mediante entrevistas, para favorecer la participación de personas analfabetas o con bajo nivel de escolaridad.

Los datos se recogieron en un modelo elaborado para captar la información sociodemográfica de los entrevistados, los datos necesarios para calcular el índice de Barthel (24) y las respuestas a las pre- guntas de las escalas del bienestar psicológico de Ryff (25). Entre la información sociodemográfica recabada se encontraba: el sexo, la edad, el nivel de estudios (sin estudios, estudios básicos y estudios avanzados o universitarios), si realizaba alguna actividad remunerada $\mathrm{y}$ el nivel de ingresos.

\section{Instrumentos empleados}

El índice de Barthel permite hacer la valoración funcional de los ancianos a partir de su capacidad para realizar las ABVD $(26,27)$ y tiene una alta validez concurrente con otras escalas similares (28). Se utilizó la versión en español validada por Baztán y colaboradores (29), de uso libre en investigaciones. Esta escala evalúa 10 áreas: alimentación, baño, vestido, aseo personal, continencia urinaria, continencia fecal, uso del retrete, traslados, deambulación y uso de escaleras, y se evalúa mediante una escala de 0 a 100 puntos.

La versión original de las escalas del bienestar psicológico de Ryff (25) se ha empleado en una gran cantidad de investigaciones en diversos países (19, 20, 30-32), y en español se ha empleado para investigaciones realizadas en España (33-35). En la presente investigación se utilizó la versión de 84 ítems, utilizada en el contexto español con resultados moderadamente satisfactorios (36). Las respuestas se dan en una escala entre 1 (totalmente en desacuerdo) y 6 (totalmente de acuerdo). Los ítems se agrupan en seis factores (14 ítems por factor): a) autonomía: independiente y autodeterminado, capaz de resistir presiones sociales para pensar y actuar de cierto modo, regula la conducta internamente y realiza autoevaluaciones desde estándares personales; b) dominio del ambiente: posee sentido de dominio y competencia para controlar el ambiente, controla múltiples conjuntos de actividades externas, hace un uso efectivo de las oportunidades del contexto y es capaz de elegir o crear contextos adaptados a sus necesidades y valores personales; c) crecimiento personal: mantiene el sentimiento de desarrollo continuado y de mejora a lo largo del tiempo, ve el yo en expansión y crecimiento, está abierto a nuevas experiencias, tiene sentido de desarrollo del propio potencial y de cambios hacia formas de ser que reflejen más autoconocimiento y efectividad; d) relaciones positivas con otros: mantiene relaciones interpersonales cercanas, satisfac- torias y de mutua confianza, se preocupa por el bienestar de otros, es capaz de desarrollar empatía, afecto, intimidad y desde la comprensión del dar y tomar de las relaciones humanas; e) autoaceptación: posee una actitud positiva hacia sí mismo, reconoce y acepta los múltiples aspectos del yo -incluido lo negativo y lo positivo-, mantiene un sentimiento positivo hacia lo ya vivido; y f) propósito en la vida: tiene metas en la vida y sentido de dirección, mantiene un sentimiento de significado en el presente y en el pasado, tiene creencias que proporcionan significado a la vida.

\section{Análisis estadísticos}

Para identificar las relaciones entre las variables de interés se probaron tres modelos de ecuaciones estructurales multifactoriales. Se consideró que las características sociodemográficas analizadas pueden afectar tanto a la salud física como al bienestar. Al tratarse de adultos mayores, el deterioro físico que produce alguna discapacidad se acelera $\mathrm{y}$, por tanto, constituye un antecedente del bienestar psicológico, por lo que en el modelo se utilizó como variable mediadora entre los factores sociodemográficos y el bienestar. Esto está respaldado por las teorías del ciclo vital, según las cuales las influencias normativas debidas a la edad y sus aspectos físico-biológicos reaparecen en las últimas etapas del desarrollo, lo que las determinan (37).

En la figura 1 se muestran los modelos teóricos alternativos probados a partir de la secuencia teórica descrita más arriba, pero con modificaciones según los factores latentes que podrían influir en las medidas del bienestar. Así, en el modelo 1 se propone un factor común latente para las seis medidas del bienestar, a partir de las sugerencias de un revisor y modelos confirmatorios de validación de las escalas de Ryff (30). El modelo 2 sigue una lógica similar y trata las medidas del bienestar de forma latente, pero las seis medidas del bienestar se dividen, según los resultados de la autora del cuestionario (20), en dos factores latentes: bienestar psicológico y bienestar subjetivo. Finalmente, el modelo 3 no propone factores latentes para las seis medidas del bienestar, dado que los estudios de validez factorial de las escalas no son determinantes en este sentido (20, 30, 35). El modelo 1 mostró la mayor parsimonia, ya que resume las relaciones de 
las medidas del bienestar en un único factor, seguido de los modelos 2 y 3 . Este último resultó ser el más complejo desde el punto de vista del análisis estadístico.

La información se procesó mediante el paquete estadístico SPSS v. 14. Los modelos estructurales se estimaron mediante el enfoque de máxima verosimilitud. Debido al fuerte alejamiento de la normalidad multifactorial en los indicadores (coeficiente multifactorial de Mardia: 24,84$)$ y a que algunas de las variables exógenas de predicción - como el nivel de estudios y los ingresos económicos- eran ordinales, se aplicaron correcciones robustas según Satorra-Bentler (38) en las pruebas de significación, los índices de ajuste y los errores estándar. Las correcciones robustas, recomendadas en situaciones como esta, se pudieron realizar gracias al considerable tamaño de la muestra (39). El ajuste de los modelos confirmatorios $(40,41)$ se evaluó mediante el índice de adecuación comparativo y los índices de bondad de ajuste simple y ajustado (valores de 0,9 o superiores indican que se puede aceptar el modelo); el error se cuantificó mediante la raíz del residuo estandarizado cuadrático medio y la raíz media cuadrática del error de aproximación (valores menores de 0,08 indican que se puede aceptar el modelo). En la evaluación del ajuste de los modelos también se empleó la prueba de la $\chi^{2}$. Para estimar los modelos estructurales se utilizó el programa EQS v. 6.1 (42).

\section{RESULTADOS}

La media de la edad en la muestra estudiada fue de 73,8 años (mínima: 65; máxima: 103; desviación estándar: 7,22); casi la mitad $(54,3 \%)$ eran mujeres y la mayoría de los participantes $(76,6 \%)$ estaban casados. En total, 35,9\% de los adultos mayores estudiados no tenía estudios, 51,6\% tenía estudios básicos y el $12,5 \%$ restante tenía estudios secundarios o universitarios; $94,3 \%$ tenía hijos y 38,7\% realizaba algún tipo de actividad remunerada. En la muestra, 56,0\% ganaba al mes aproximadamente US\$ 86,00; 25,7\% ganaba entre US $\$ 87,00$ y US\$ 143,00 ; $10,0 \%$ entre US\$ 144,00 y US\$ 286,00; y solo $8,3 \%$ ganaba más de US\$ 286,00.

En el cuadro 1 se muestran las medias y las desviaciones estándar de las variables cuantitativas empleadas en el modelo. De los tres modelos alternativos presentados (figura 1), el modelo 3 fue el

FIGURA 1. Modelos teóricos probados para predecir el bienestar y la capacidad para realizar actividades básicas de la vida diaria (ABVD)

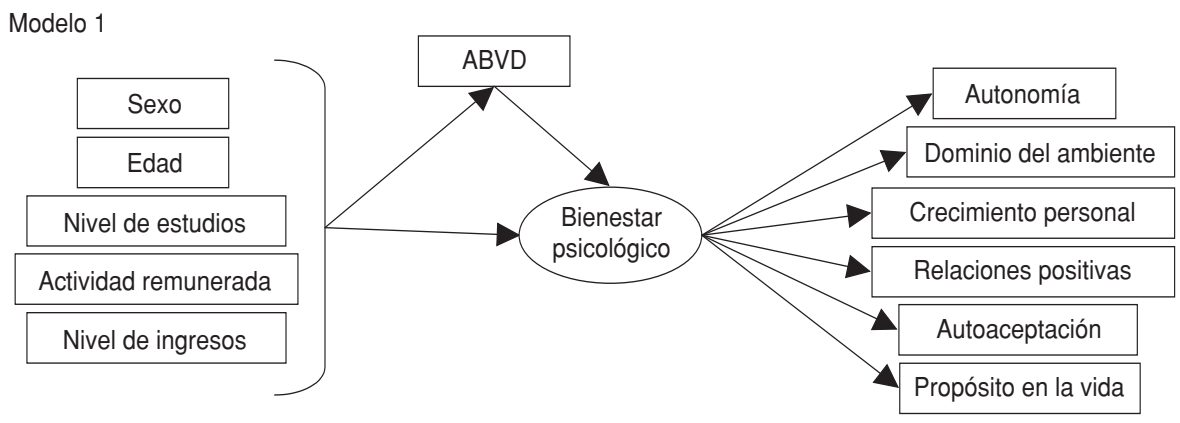

Modelo 2

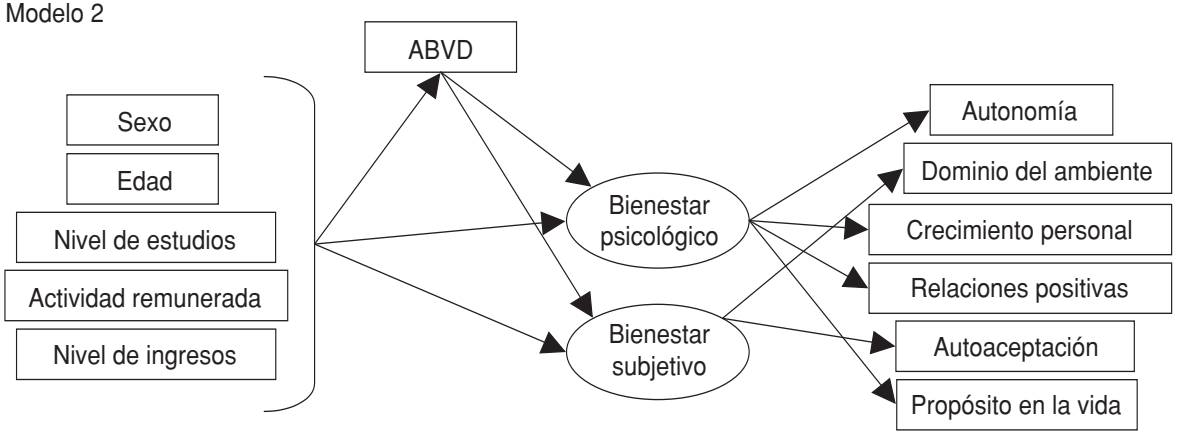

Modelo 3

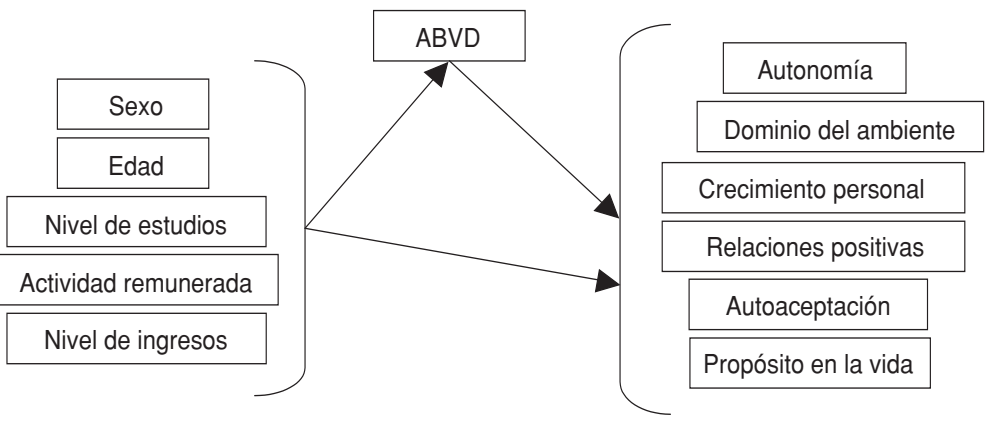

CUADRO 1. Características de la muestra estudiada según las dimensiones de las escalas del bienestar y la capacidad para realizar actividades básicas de la vida diaria (ABVD)

\begin{tabular}{lrc}
\hline \multicolumn{1}{c}{ Dimensiones } & Media & Desviación estándar \\
\hline ABVD & 91,18 & 16,15 \\
Autonomía & 3,66 & 0,66 \\
Dominio del ambiente & 3,79 & 0,68 \\
Crecimiento personal & 3,98 & 0,67 \\
Relaciones positivas & 3,78 & 0,62 \\
Autoaceptación & 3,74 & 0,63 \\
Propósito en la vida & 3,70 & 0,64 \\
\hline
\end{tabular}

único que presentó índices de ajuste satisfactorios por encima de los puntos de corte (cuadro 2). Este modelo no propone ningún factor latente a partir de los seis componentes del bienestar $\mathrm{y}$, por tanto, permite estimar el efecto de los factores sociodemográficos y de la capa- cidad para realizar ABVD (índice de Barthel) de manera directa a partir de las seis medidas del bienestar.

El modelo estructural final mantuvo una serie de relaciones estandarizadas (ilustradas mediante flechas en la figura 2) que no se pueden eliminar sin empeo- 
CUADRO 2. Ajuste de los modelos estructurales probados según diversas pruebas ${ }^{\mathrm{a}}$

\begin{tabular}{|c|c|c|c|c|c|c|c|c|}
\hline \multirow[b]{2}{*}{ Modelos estructurales } & \multicolumn{3}{|c|}{ Prueba de la ji al cuadrado } & \multicolumn{5}{|c|}{ Índices de ajuste ${ }^{b}$} \\
\hline & $\chi^{2}$ & Grados de libertad & $P$ & $\mathrm{CFI}$ & RMSEA & GFI & AGFI & SRMR \\
\hline Modelo 1 & 867,92 & 70 & $<0,01$ & 0,850 & 0,090 & 0,906 & 0,858 & 0,080 \\
\hline Modelo 3 & 209,825 & 34 & $<0,01$ & 0,966 & 0,052 & 0,977 & 0,947 & 0,061 \\
\hline
\end{tabular}

a En los modelos se eliminaron las relaciones no significativas $(P>0,05)$, por ser irrelevantes (nulas en la población).

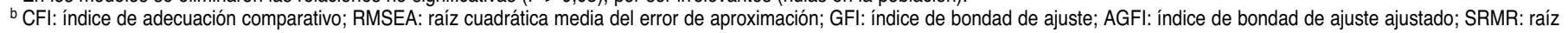
del residuo estandarizado cuadrático medio.

FIGURA 2. Modelo estructural final para predecir la capacidad de realizar actividades básicas de la vida diaria (ABVD) y el bienestara

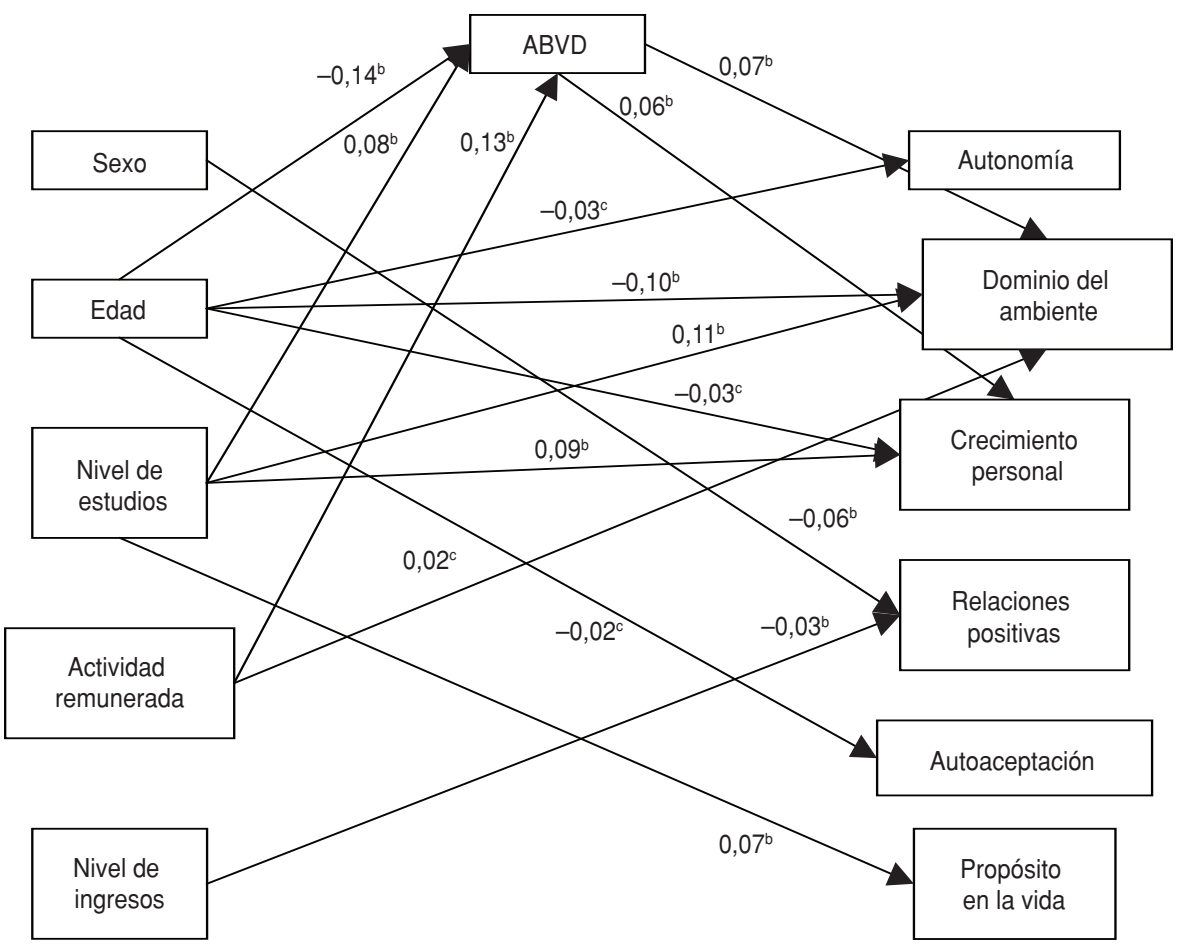

a Las flechas indican las relaciones estandarizadas y las cifras indican las estimaciones de los efectos de unas variables sobre otras, redondeadas al segundo decimal. Las relaciones entre las medidas del bienestar no se muestran en aras de simplificar la figura.

${ }^{b}$ Relación significativa $(P \leq 0,05)$

${ }^{c}$ Relación no significativa $(P>0,05)$.

rar de forma significativa el ajuste del modelo. Por lo tanto, esas relaciones son relevantes en la población estudiada. En la figura 2 no se indican las posibles relaciones de predicción de la capacidad para realizar las ABVD y el bienestar, porque no resultaron estadísticamente significativas, es decir, se pueden considerar nulas en esta población.

Estos resultados muestran claramente que el efecto de los factores sociodemográficos sobre la capacidad para realizar las ABVD era muy pequeño y se concentró en pocas variables. En el caso de la autonomía y de la autoaceptación, ninguna de las variables sociodemográficas o de predicción, o el nivel de ABVD tuvieron una influencia estadísticamente significativa $(P>0,05)$. Por tanto, la predicción resultó totalmente externa al modelo.

Se observó que el sexo presentó una relación significativa solamente con las relaciones positivas con otros y las mujeres presentaron un nivel mayor en este componente del bienestar. Por su parte, las relaciones entre la edad, por una parte, y la capacidad para realizar las ABVD y el dominio del ambiente, por la otra, fueron ligeramente negativas, lo que indica que con la edad, aumentó la dependencia para realizar las actividades diarias y se redujo ligeramente el dominio del ambiente. El nivel de estudios fue el indicador que más relaciones mostró con las variables endógenas (a predecir) del modelo. Así, las relaciones fueron siempre positivas, tanto con el nivel de dependencia (para las ABVD) como con las dimensiones propias del bienestar subjetivo (dominio del ambiente) y psicológico (crecimiento personal y propósito en la vida). Por su parte, el efecto de realizar actividades remuneradas y del nivel de ingresos fue mucho más modesto, aunque positivo, en el primer caso sobre las ABVD y en el segundo sobre las relaciones positivas con otros. El nivel de dependencia para realizar las ABVD parece haber afectado principalmente, y de forma positiva, al dominio del ambiente y al crecimiento personal.

Se debe subrayar que la capacidad predictiva del modelo para las variables endógenas fue débil. Las varianzas explicadas de los componentes del bienestar fueron bajas: $0,1 \%$ para la autonomía; $3,3 \%$ para el dominio del ambiente; $1,3 \%$ para el crecimiento personal; $0,4 \%$ para las relaciones positivas con otros; $0,01 \%$ para la autoaceptación; y $0,5 \%$ para el propósito en la vida. La varianza de los factores sociodemográficos explicó 5,1\% de la varianza del nivel de ABVD de los adultos mayores dominicanos.

Adicionalmente, los componentes del bienestar del modelo presentaron múltiples correlaciones entre sí, lo que indica que lejos de ser factores independientes, todos guardaban una estrecha relación (cuadro 3). Estas correlaciones no se muestran en el modelo de la figura 2 para evitar hacerla más engorrosa, aunque forman parte de ese complejo sistema de interrelación.

Por último, se encontró una correlación negativa entre la realización de actividades remuneradas y la edad $(r=-0,2$; $P<0,01)$ y una relación positiva - ya bien conocida- entre el nivel de estudios y los ingresos $(r=0,44 ; P<0,01)$. 
CUADRO 3. Correlaciones entre las dimensiones de las escalas del bienestar

\begin{tabular}{|c|c|c|c|c|c|c|}
\hline Dimensiones & Autonomía & $\begin{array}{c}\text { Dominio } \\
\text { del ambiente }\end{array}$ & $\begin{array}{c}\text { Crecimiento } \\
\text { personal }\end{array}$ & $\begin{array}{c}\text { Relaciones } \\
\text { positivas }\end{array}$ & Autoaceptación & $\begin{array}{l}\text { Propósito } \\
\text { en la vida }\end{array}$ \\
\hline Autonomía & -- & & & & & \\
\hline Dominio del ambiente & 0,681 & - & & & & \\
\hline Crecimiento personal & 0,638 & 0,716 & - & & & \\
\hline Relaciones positivas & 0,660 & 0,705 & 0,677 & - & & \\
\hline Autoaceptación & $N A^{a}$ & 0,688 & 0,687 & 0,735 & - & \\
\hline Propósito en la vida & 0,679 & 0,725 & 0,703 & 0,707 & 0,734 & - \\
\hline
\end{tabular}

a NA: no aplica. La correlación entre las dimensiones autonomía y autoaceptación no se estimó por no resultar significativa en el modelo inicial $(P>0,05)$.

\section{DISCUSIÓN}

A partir del modelo de ecuaciones estructurales se puede llegar a algunas conclusiones. La primera es el pequeño poder predictivo de las variables sociodemográficas, tanto sobre la capacidad de realizar las ABVD como sobre el bienestar. Esto es muy importante, ya que, si bien las relaciones encontradas son similares a las halladas en estudios realizados en América del Norte y Europa, no resultaron tan fuertes y generalizadas; además, las varianzas explicadas fueron ligeramente menores que las de esos estudios (43). En efecto, los estudios realizados en Europa y los Estados Unidos de América muestran una mayor capacidad predictiva en un mayor número de componentes del bienestar. Una segunda conclusión es que las variables concretas más afectadas por las características sociodemográficas fueron, precisamente, la capacidad para realizar las ABVD y el dominio del ambiente. Estas dos variables se encuentran relacionadas entre sí, dado que difícilmente se puede percibir un adecuado dominio del ambiente cuando se tienen dificultades para realizar las ABVD.

En relación con los factores sociodemográficos, parece claro que existe una relación entre el género y las relaciones sociales positivas, lo que puede deberse a que la mayor parte de los esfuerzos para mantener las relaciones familiares y del hogar recae en las mujeres. Se observó, además, una clara orientación de las mujeres hacia los hijos como proveedores de apoyo, con el consiguiente aumento de las relaciones positivas (44). Esto confirma estudios previos realizados en España mediante modelos de ecuaciones estructurales que indican que el género solamente se relaciona con la dimensión de las relaciones positivas (45).

Sin embargo, otros autores han encontrado que los hombres tienen una percepción de su salud significativamente mejor que las mujeres, algo similar a lo que ocurre con respecto a la capacidad para realizar las $\operatorname{ABVD}(4,5,46,47)$. En el presente estudio, esta relación no fue significativa en ningún caso, aunque se debe tener en cuenta que al aumentar la esperanza de vida en las mujeres aumenta también el tiempo que vivirán con dependencia funcional (48). Dado que el modelo estructural presenta una estimación del efecto del género controlado por la edad, las diferencias en la capacidad para realizar las ABVD entre los hombres y las mujeres puede deberse más al efecto de la edad que al propio género.

Algunos trabajos realizados en México a partir de los datos de la encuesta SABE indican que a medida que aumenta la edad se incrementa la proporción de personas que tienen dificultades para desempeñar las $\operatorname{ABVD}(4,49,50)$. Alrededor de $20 \%$ de los adultos mayores entrevistados tenía dificultades con las ABVD y esa cifra puede aumentar a $26 \%$ entre los adultos de más de 70 años (51). Como referencia, en España, el grupo de más edad presenta mayores limitaciones para realizar las ABVD, especialmente a partir de los 80 años $(5,52)$. Si bien se observa un retraso en la aparición de las dificultades para realizar las ABVD en adultos mayores de menos de 83 años, a mayores edades se produce una inversión de esta tendencia (53). Esto respalda la ligera relación negativa mostrada por el modelo estructural presentado.

Según aumentó la edad se redujo la puntuación en la dimensión dominio del ambiente, que tiene que ver con el uso efectivo de las oportunidades y la creación de contextos adaptados. Se debe tener en cuenta que el mayor uso de estrategias acomodativas por parte de los adultos mayores implica que la persona modifique sus objetivos, ya sea reduciendo su nivel de aspiración, desechando ciertos objetivos para establecer otros o estableciendo parámetros alternativos de comparación para evaluar su situación actual (54). La acomodación no implica una acción intencional dirigida a una meta, sino un proceso adaptativo que se produce de forma automática (36). La capacidad para realizar las ABVD y el dominio del ambiente también mostró una relación significativa con la edad. Así, parece ser que las personas que muestran una mejor valoración funcional poseen un adecuado sentido de dominio del ambiente y son capaces de elegir o crear diversos contextos adaptados a sus necesidades y valores personales. Los resultados obtenidos demostraron que la edad afecta a las dimensiones de autonomía y dominio del ambiente, mientras que estudios realizados en países anglosajones (43) mostraron un efecto generalizado de la edad sobre los componentes del bienestar, tanto psicológico como subjetivo $(P<0,01)$, aunque estos efectos tampoco fueron especialmente grandes.

El grupo con mayor nivel de estudios presentó una mayor satisfacción con la vida, al igual que otras poblaciones en las que se observaron relaciones significativas y positivas $(P<0,01)$ del nivel educativo con el grado de satisfacción y el bienestar subjetivo (55) y diferencias de más de cinco puntos entre la calificación de la satisfacción de personas con educación universitaria y la de personas sin estudios $(P=0,03)(56)$. Al igual que en la presente investigación, otros autores han observado un efecto sistemático y positivo del nivel de estudios sobre la capacidad de realizar ABVD en América Latina y el Caribe (7). En estudios de predicción de los componentes del bienestar, el nivel de estudios se ha asociado positivamente con los componentes del bienestar $(4,43)$. En el presente estudio se observó una relación entre las dos medidas del bienestar psicológico y las dimensiones crecimiento personal, propósito en la vida y dominio del ambiente.

El presente trabajo es una de las primeras investigaciones sobre bienestar y ABVD en adultos mayores dominicanos y 
se ha basado en una muestra representativa según los requisitos estadísticos más estrictos. Sin embargo, al analizar estos resultados se debe tener en cuenta el carácter transversal de la investigación, lo que hace difícil establecer una dirección de causalidad en las relaciones encontradas.

En conclusión, los factores sociodemográficos con capacidad de predicción más importantes en esta muestra fueron la edad - relacionada negativamente con algunas dimensiones del bienestar y la capacidad para realizar las ABVD-y el sexo femenino - que se relacionó negativamente con la dimensión de relaciones positivas con otros-. El nivel de estudios influyó positivamente en el dominio del ambiente, el crecimiento personal, el propósito en la vida y la capacidad para realizar las ABVD.

Estos resultados demuestran la importancia de destinar recursos a este sector de la población, menor en número, pero tan relevante como los niños y los jóvenes, principales receptores de los esfuerzos en salud pública y educación en la
República Dominicana. Los modelos validados en este trabajo brindan una valiosa información, necesaria para desarrollar políticas orientadas a dos niveles de acción complementarios: la promoción de la autonomía de los adultos mayores y el aumento de la cobertura de los sistemas de enseñanza formal. Estos resultados respaldan la conveniencia de aumentar las inversiones en políticas sociales y de diseñar investigaciones que contribuyan a lograr el objetivo básico de mejorar la calidad de vida de toda la población.

\section{REFERENCIAS}

1. Comisión Económica para América Latina y el Caribe. América Latina y el Caribe: el envejecimiento de la población, 1950-2050. Santiago: CEPAL; 2003.

2. Comisión Económica para América Latina y el Caribe. Los adultos mayores en América Latina y el Caribe. Datos e indicadores. Santiago: CEPAL; 2002.

3. Huenchuan S, Paredes M, González D. Escenarios futuros de políticas de vejez en República Dominicana: la persistente demanda por derechos básicos. Santiago: CEPAL; 2006.

4. Dorantes-Mendoza G, Ávila-Funes JA, MejíaArango S, Gutiérrez-Robledo LM. Factores asociados con la dependencia funcional en los adultos mayores: un análisis secundario del Estudio Nacional sobre Salud y Envejecimiento en México, 2001. Rev Panam Salud Publica. 2007;22:1-11.

5. Abellán A, Esparza C. Las personas mayores con dependencia. En: Puyol R, Abellán A, coords. Envejecimiento y dependencia. Una mirada al panorama futuro de la población española. Madrid: Mondial Asístanse; 2006. Pp. 53-72.

6. Instituto de Mayores y Servicios Sociales. Libro blanco de la dependencia. Madrid: Ministerio de Trabajo y Asuntos Sociales; 2005.

7. Reyes-Ortiz CA, Ostir GV, Peláez M, Ottenbacher KJ. Cross-national comparison of disability in Latin American and Caribbean persons aged 75 and older. Arch Gerontol Geriatr. 2006;42:21-33.

8. Instituto Nacional de Estadística. Encuesta sobre discapacidades, deficiencias y estado de salud 1999. Resultados detallados. Madrid: INE; 2002.

9. Organización Panamericana de la Salud. Salud bienestar y envejecimiento (SABE) en América Latina y el Caribe. Informe preliminar. Washington, D.C.: OPS; 2001.

10. Peláez M, Pratts O, Palloni A, Hennis AJ, Ham-Chande R, León Díaz EM, et al. Encuesta Salud, Bienestar y Envejecimiento (SABE): metodología de la encuesta y perfil de la población estudiada. Rev Panam Salud Publica. 2005;17:307-22.

11. Menéndez J, Guevara A, Arcia N, León Díaz EM, Marín C, Alfonso JC. Enfermedades crónicas y limitación funcional en adultos mayores: estudio comparativo en siete ciudades de América Latina y el Caribe. Rev Panam Salud Publica. 2005;17:353-61.
12. Consejo de Europa. Recomendación No. R (98) 9 del Comité de Ministros a los Estados Miembros relativa a la dependencia [sitio en Internet]. Madrid: Instituto de Mayores y Servicios Sociales; 1998. Hallado en http:// www.imsersomayores.csic.es/documentos/ documentos/consejoeuropa-rec989-01.pdf. Acceso el 14 de agosto de 2009.

13. Organización Mundial de la Salud. Clasificación Internacional del Funcionamiento, la Discapacidad y la Salud (CIF). Ginebra: OMS; 2001.

14. Rowe JW, Kahn RL. Human aging: usual and successful aging. Science. 1987;237:143-9.

15. Rowe JW, Kahn RL. Successful aging. Gerontologist. 1997;37:433-40.

16. Lyubomirsky S, King L, Diener E. The benefits of frequent positive affect: does happiness lead to success? Psychol Bull. 2005;131:803-55.

17. Vecina ML. Emociones positivas. Papeles Psicólogo (España). 2006;1:9-17.

18. Ostir G, Markides K, Black S, Goodwin J. Emotional well-being predicts subsequent functional independence and survival. J Am Geriatr Soc. 2000;48:473-8.

19. Ryff $C D$, Keyes $C$. The structure of psychological well-being revisited. J Pers Soc Psychol. 1995;69:719-27.

20. Keyes C, Shmotkin D, Ryff CD. Optimizing well-being: the empirical encounter of two traditions. J Pers Soc Psychol. 2002;82:1007-22.

21. Diener E, Lucas RE. Personality and subjective well-being. In: Kahneman D, Diener E, Schwarz N, eds. Well-being: the foundations of hedonic psychology. New York: Russell Sage; 1999. Pp. 213-27.

22. Villar F, Triadó C, Resano CS, Osuna MJ. Bienestar, adaptación y envejecimiento: cuando la estabilidad significa cambio. Rev Multidisciplinar Gerontol. 2003;13:152-62.

23. Diener E. Subjective well being: the science of happiness, and a proposal for a national index. Am Psychol. 2000;55:34-43.

24. Mahoney FI, Barthel DW. Functional evaluation: the Barthel index. Md State Med J. 1965; $14: 61-5$.

25. Ryff CD. Happiness is everything: or is it? Explorations on the meaning of psychological well-being. J Pers Soc Psychol. 1989;57:1069-81.

26. Shah S, Vanclay F, Cooper B. Improving the sensitivity of the Barthel index for stroke rehabilitation. J Clin Epidemiol. 1989;4:703-9.
27. Cid-Ruzafa J, Damián-Moreno J. Valoración de la discapacidad física: el índice de Barthel. Rev Esp Salud Publica. 1997;71:127-37.

28. Perlado F, Alastuey C, Espinosa C, Clemencia M, Ochoa P, Domingo D. Valoración del estado funcional en ancianos hospitalizados: la escala Plutchik. Med Clin. 1996;107:45-9.

29. Baztán JJ, Pérez del Molino J, Alarcón T, San Cristóbal E, Izquierdo G, Manzarbeitia J. Índice de Barthel: instrumento válido para la valoración funcional de pacientes con enfermedad cerebrovascular. Rev Esp Geriat Gerontol. 1993;28:32-40.

30. Van Dienrendonck D. The construct validity of Ryff's Scale of psychological well-being and its extension with spiritual well-being. Pers Individ Dif. 2005;36:629-44.

31. Abbott RA, Ploubidis GB, Huppert FA, Kuh D, Wadsworth MEJ, Croudace TJ. Psychometric evaluation and predictive validity of Ryff's psychological well-being items in a UK birth cohort sample of women. Health Qual Life Outcomes. 2006;4:76.

32. Ryff $C D$, Singer $B H$. Best news yet on the sixfactor model of well-being. Soc Sci Res. 2006; 35:1103-19.

33. Villar F, Triadó C, Solé C, Osuna MJ. La medida del bienestar en personas mayores: adaptación de la escala de Ryff. Rev Psicol Gen Apl. 2005;58:347-64.

34. Triadó C, Villar F, Solé C, Celdrán M. Construct validity of Ryff's scale of psychological well-being in Spanish older adults. Psychol Rep. 2007;100:1151-64.

35. Tomás J, Meléndez JC, Navarro E. Modelos factoriales confirmatorios de las escalas de Ryff en una muestra de personas mayores. Psicothema. 2008;20(2):298-304.

36. Triadó MC. Envejecer en entornos rurales. Madrid: IMSERSO, Estudios I+D+I; 2003. (No. 19.)

37. Baltes PB. Theoretical propositions of life-span developmental psychology: on the dynamics between growth and decline. Dev Psychol. 1987;23:611-26.

38. Satorra A, Bentler PM. A scaled difference chi-square test statistic for moment structure analysis. Psychometrika. 2001;66:507-14.

39. Finney SJ, DiStefano C. Non-normal and categorical data in SEM. In: Hancock GR, Mueller RO, eds. Structural equation modelling: a second course. Greenwich, CO: Information Age; 2006. Pp. 269-314. 
40. Hu L, Bentler PM. Cut-off criteria for fit indexes in covariance structure analysis: conventional criteria versus new alternatives. Struct Equation Model. 1999;6:1-55.

41. Kaplan D. Structural equation modeling: foundations and extensions. Thousand Oaks, CA: Sage; 2000.

42. Bentler PM. EQS program manual. Encino, CA: Multivariate Software; 2005.

43. Ryff CD. Optimizing well-being: the empirical encounter of two traditions. J Pers Soc Psychol. 2002;82:1007-22.

44. Meléndez JC, Tomás JM, Navarro E. Análisis de las redes sociales en la vejez a través de la entrevista Manheim. Salud Publica Mex. 2007; 49:408-14.

45. Meléndez JC, Tomás JM, Oliver A, Navarro E. Psychological and physical dimensions explaining life satisfaction among the elderly: a structural model examination. Arch Gerontol Geriatr. 2009:48:291-5.

46. Organización Mundial de la Salud. Encuesta multicéntrica Salud Bienestar y Envejecimiento (SABE) en América Latina y el Caribe. Washington, D.C.: OPS; 2001.
47. Fernández-Ballesteros R, Zamarrón MD, Diez J, de Juan M, Montero P, López-Bravo MD, et al. Estudio longitudinal sobre envejecimiento. Madrid: IMSERSO, Estudios I+D+I; 2005. (No. 38.)

48. Jentoft-Cruz AJ. Asistencia médica al paciente geriátrico. Medicine. 2003;8:5772-7.

49. Albala C, Lebrão ML, León EM, Ham-Chande R, Hennis AJ, Palloni A, et al. Encuesta Salud, Bienestar y Envejecimiento (SABE): metodología de la encuesta y perfil de la población estudiada. Rev Panam Salud Publica, 2005;17: 307-22.

50. Barrantes-Monge M, García-Mayo EJ, GutiérrezRobledo LM, Miguel-Jaimes A. Dependencia funcional y enfermedades crónicas en ancianos mexicanos. Salud Publica Mex. 2007;49:459-66.

51. Comisión Económica para América Latina y el Caribe. Las personas mayores en América Latina y el Caribe: diagnóstico sobre la situación y las políticas. Santiago: CEPAL-ONU; 2003.

52. Yanguas JJ. Análisis de la calidad de vida relacionada con la salud en la vejez desde una perspectiva multidimensional. Madrid: IMSERSO; 2006.
53. Otero A, Zunzunegui MV, Rodríguez-Laso A, Aguilar MD, Lázaro P. Volumen y tendencia de la dependencia asociada al envejecimiento en la población española. Rev Esp Salud Publica. 2004;78:201-13.

54. Navarro E, Meléndez JC, Tomás JM. Análisis del bienestar en la vejez según la edad. Rev Esp Geriatr Gerontol. 2008;43:90-5.

55. Pinquart $\mathrm{M}$, Sorensen $\mathrm{S}$. Gender differences in self concept and psychological well being in old age: a meta-analysis. J Gerontol. 2001;56: 195-216.

56. Subas F, Hayran O. Evaluation of life satisfaction index of the elderly people living in nursing homes. Arch Gerontol Geriatr. 2005;41: 3-29.

Manuscrito recibido el 23 de abril de 2008. Aceptado para publicación, tras revisión, el 9 de enero de 2009.
ABSTRACT

\section{Structural equation model for predicting well-being and functional dependency of the elderly in the Dominican Republic}

Objective. To produce a multivariate structural model that explains functional dependence and well-being in terms of certain related sociodemographic factors among elderly adults in the Dominican Republic.

Methods. A cross-sectional study that surveyed a sample of 1397 individuals 65 or more years of age, representing the Dominican Republic's elderly population. Those surveyed were asked for sociodemographic information (sex, age, level of education, employment status, and income level), data needed to determine their Barthel Index, and responses to questions from Ryff's Scale of Psychological Well-being. Three structural equation models were used to identify relationships among the key variables.

Results. The most fitting structural adjustment model linked sociodemographics to the ability to perform basic activities of daily living (BADL) and the Ryff's Scale of Psychological Well-being, without suggesting latent factors. With regard to endogenous variables, the model's predictive power was weak. Age was negatively associated with some areas of well-being and with the ability to perform BADL; sex was related to the area of positive relationships with others; and educational level positively influenced environment, personal growth, and having a sense of purpose, as well as the ability to perform BADL.

Conclusions. The proven models provide information necessary for developing policies aimed at two levels of coordinated action: promoting autonomy among elderly adults and expanding enrollment in formal education programs. These results support efforts to increase investment in social policies.

Key words Health of the elderly; quality of life; mathematical models; Dominican Republic. 